Stoa

Vol. 2, No. 4, 2011, pp.79-94

ISSN:2007-1868

\title{
APUNTES HERMENÉUTICOS A LA DIFERENCIA EN EL USO DE LA ANALOGÍA EN LEIBNIZ Y LA ANALOGÍA EN KANT
}

\author{
Rubén MendozA. \\ Facultad de Filosofía y Letras \\ Universidad Autónoma de México \\ A Julia Barajas \\ rubmendozza@yahoo.it
}

RESUMEN: Uno de los temas centrales en hermenéutica es, sin duda, la analogía. Este concepto se nos muestra como un elemento constitutivo en la explicación del acto interpretativo y, sobre todo, en la construcción de conocimiento. Tanto Leibniz como Kant, ocuparon la analogía para elaborar algunas explicaciones filosóficas con relación al entendimiento humano, donde el primero ocupa la analogía de proporción y el segundo la analogía de atribución. Con estos apuntes, se quiere destacar la importancia de esta categoría como una de las más importantes entro de la Historia del pensamiento Occidental.

PALABRAS CLAVE: analogía, interpretación, proporción, atribución, conocimiento.

noindent SUMMARY: One of the central themes in hermeneutics is without doubt the analogy. This concept appears to us as a constitutive element in the explanation of the interpretive act, and especially in the construction of knowledge. Both Leibniz and Kant, took the analogy to make some philosophical explanations in relation to human understanding, and Leibniz took the analogy of proportion and Kant use the analogy of attribution. With these notes, seeks to highlight the importance of this category as one of the largest entered in the history of universal knowledge. noindent KEY WORDS: analogy, interpretation, proportion, attribution, knowledge.

\section{Presentación}

Al hablar de analogía siempre hacemos referencia a lo semejante y a lo diferente, como un modo de interpretar la realidad. En ello, la analogía es en sí misma analógica, utiliza una hermenéutica con sus propios límites y condiciones simbólicas, y esto por el hecho mismo de la interpretación de lo semejante en lo diferente y viceversa, de lo diferente con relación a lo semejante, dados 
los contextos y las posibles conjeturas entre los sentidos y los referentes "originarios” de un texto. No obstante, “(...)la analogía está entre la identidad y la diferencia" (Beuchot., 2009, p. 7), resaltar el está entre como un puente es ya analogía, pues es su trabajo principal, crear puentes entre las posibles referencias contextuales y los sentidos textuales a partir de la intencionalidad (Mendoza, 2010) del autor en cuestión. Este "está entre" es el que nos enfrenta al horizonte de lo "alcanzable", poco, pero suficiente, de las verdades condicionadas por el devenir histórico y concreto cultural de un escrito en general. En esto, existen dos autores que utilizaron la analogía en sus escritos, tanto para ejemplificar como para generar conocimiento en áreas específicas.

Nos referimos a Gottfried Wilhelm Leibniz e Immanuel Kant. Del primero extraeremos la concepción de analogía en tres ensayos que se titulan: 1.demostraciones de las proposiciones primarias de 1671-72, 2.- la profesión de fe del filósofo de 1673 y, 3.- Verdades necesarias y contingentes de 1686. Del segundo utilizaremos el parágrafo 58 de los Prolegómenos a toda metafísica del porvenir de 1783 (del futuro en la traducción de Besteiro) y la Crítica de la razón pura de 1781, en la Analítica trascendental, libro segundo, capítulo segundo, sección tercera: Analogías de la experiencia. Hacemos mención de los ensayos y de los apartados que utilizaremos más para justificar y al mismo tiempo delimitar este trabajo, pues no pretendemos hacer "una" interpretación absoluta y universal del trabajo de estos dos grandes autores, lo que se quiere es indagar acerca de la posible utilización conceptual de construcción de conocimiento (sí es que existe) entre la idea de armonía preestablecida (que es la analogía por excelencia de Leibniz) y la orientación de conocer trascendental (en Kant) de la analogía. ${ }^{1}$

Antes de entrar en materia, hablemos un poco de qué entendemos por analogía. Podríamos definir la analogía como una figura retórica, o como un instrumento lingüístico, o como una forma interpretativa de construcción explicativa, la cual siempre tiende a la semejanza y a la diferencia. Teofrasto menciona que "hay que intentar poner en claro de la manera que sea, bien por analogía, bien por otra clase de semejanza, cuál es esa entidad o, cuáles si son varias (IV). Santo Tomás dice que la analogía siempre tiende hacia algo distinto, y eso de distinto hace de la analogía, una manera específica de

\footnotetext{
${ }^{1}$ También utilizaremos algunos otros textos para ejemplificar o profundizar en el concepto.
} 
utilidad (utens, instrumento) y de referente teórico (docens, doctrina) al momento de interpretar (Cfr., Beuchot, 2009). Se convierte en un puente, en un "estar entre", a partir de un proceso que hemos llamado de desambigüación, el cual, no es otra cosa que tratar de desenmarañar las intencionalidades del autor, del lector y del texto, y referir, según sea el caso, a la "ambigüedad" de las posibles correctas interpretaciones.

Así, en el fragmento, la analogía alude a la condición de posibilidad de "acercarnos" de manera prudente a los posibles sentidos originarios y auténticos de un texto. Ir hacia las causas primeras o principios como menciona Aristóteles en la Física (I, 184a) y en la Metafísica (I, 25), es andar un camino, es recorrer cada uno de los puntos de la realidad, esos fragmentos que nos permiten conocer el todo de forma reducida, pero suficiente. Como menciona Beuchot (2002) "(...) con un fragmento nos da el conocimiento de la totalidad, la parte nos conduce al todo, el fragmento nos lleva al conjunto". Y es precisamente lo que queremos alcanzar, una parte, esa reducción que nos otorgue ciertos límites interpretativos, sin los cuales, no podríamos construir interpretaciones. Así, la analogía en palabras de Ricoeur (2001. p. 359) hablando sobre la posición tomista comenta: “(...)el nuevo uso del concepto de analogía podía parecer justificado por el paralelismo de las situaciones iniciales de discurso. En efecto, el problema es abrirse un camino intermedio entre dos imposibilidades". Y esto es verdad en el sentido de la intermediación, como tópico medio entre dos puntos que se alejan o se acercan de repente como meras imposibilidades. Analógicamente hablando, esto no querría decir que la analogía sólo es un mero "pasamanos" abierto a cualquier cosa, sino todo lo contrario, también constituye, en ella misma, su propio límite, es decir, es autorreferente, por ser intermediación, alude a sí misma y busca en sí misma su explicación.

Hacer analogías no significa entrar en el mundo de la ocurrencia, más aún, indica simbólicamente algunos alcances del trabajo hermenéutico. Por ello decíamos más arriba que la analogía es hermenéutica (como arte como ciencia), pues nos introduce en las condiciones de posibilidad del texto, sin caer en un eclecticismo literalista o un escepticismo sin límites. El problema de la analogía es que, siendo ella misma autorreferente, es en sí un principio que no conlleva otro fundamento o elemento primario más que su soporte epistémico 
y metodológico, es decir, que debemos pensarla como principio sin principio, si no se nos iría al infinito (petición de un principio con base en otro principio, y así sucesivamente) y no lograríamos una traducción adecuada por estar verificando si el principio es o no es "congruente" con su estructura, por lo que estamos supeditados a su demarcación teorética.

Más fácil, la analogía es autorreferente por el simple hecho de que ella es un fundamento que nos permite construir conocimiento, más no es lo construido ni es lo por construir. Si pensamos un poco más en esto, la referencia a sí misma (lo que hemos llamado la desambigüación (Mendoza, 2010) le da sentido a la analogía, sin sentido y sin referencia una hermenéutica o cualquier figura retórica no tendría razón de ser, puesto que la interpretación sin sentido no es comprensible y sin referente no podría ser explicada. Lo implícito en la analogía como desambigüación (y esto es nuevo) no está condicionado por el aparato teórico exclusivamente, sino por la intencionalidad en el acto interpretativo. Si bien es cierto que la intencionalidad tiene "remanentes" conceptuales, esto no significa que se quede en ese plano, pues la intencionalidad no es un elemento psicológico (con contenido explícito o como propiedad de una experiencia que consiste en ser conciencia de algo como nos comenta Paredes, 2007), como tampoco es un "incidente" cognitivo, ni mucho menos un acto de voluntad cedido por designio, sino que es un modo de ser en el ser que está en el mundo, un existenciario, el primero diría yo, de todo acto de conocimiento de la realidad, fragmento dado por la analogía.

O como lo menciona Spiegelberg, al comentar sobre la teoría escolástica de Aquino, "(...) la intencionalidad significa un cierto modo de existencia” (1981 a, p. 13 cit. Paredes, 2007). Acá agregaríamos que esa existencia, recae no en las palabras como menciona Avicenas, sino que aquella es existencia por modo del ser en el mundo, no es un objeto, como tampoco podría situarse en el mero acto cognitivo (hacer conciencia de algo), sino que siendo en el mundo, el existenciario, aquello que fenomenológicamente se manifiesta en sí, como principio primario, es, de un modo u otro, la manera de ser, ese modo análogo a sí mismo. Por tanto, la intencionalidad concuerda con la analogía como desambigüación al momento exacto de aprehender eso que llamamos mundo. Sin lo cual, nuestra interpretación sería sesgada o ambivalente o incongruente. Necesitamos la analogía para tener un paralelo en el cual podamos movernos, 
tal vez un poco apretados, pero lo suficientemente abierto, para indagar nuestra actividad y la misma interpretación del texto, el contexto y la traducción implícita en el [F] acto interpretativo (Cfr., Mendoza 2008).

Ahora bien, pensamos que esta exposición sobre la analogía no está lo suficientemente aclarada, ni mucho menos acabada para agradar al lector atento, simplemente hemos querido (y la tónica va hacer la misma a lo largo del trabajo) dar una panorámica general de definición. Me dedicaré en lo que sigue, a entresacar algunas líneas de discusión, tanto con Leibniz como con Kant, sobre este punto. La pregunta guía es esta: cla analogía, puede llegar a determinar la construcción del conocimiento o simplemente es un "modelo" para interpretar lo ya construido? La respuesta posiblemente nos la otorguen estos dos autores. Divido esto por facilidad de lectura, en tres apartados: 1.- la analogía en Leibniz, 2.- la analogía en Kant y, 3.- conclusiones generales. Cabe mencionar que trazaremos la analogía como búsqueda, otros temas los dejaremos para mejor ocasión.

\section{La analogía en Leibniz.}

Gottfried Whelm Leibniz (1646-1716), no sólo entra a la Historia del pensamiento por sus aportes en filosofía, política o ciencia, sino porque es uno de esos hombres que se interesaron por el conocimiento en general, por acto de conocer, aquello que menciona Aristóteles en el libro I de la Metafísica, el hombre desea saber, y agregamos, prefiere eso que ignorar. Esto le queda muy bien a Leibniz, el cual demostró no sólo su apetito hacia los conocimientos concretos de la realidad, sino que abarcó lo abstracto y lo mecánico, el cálculo matemático (con la debida disputa con otro grande como Newton y la paternidad de lo infinitesimal) y la crítica a la metafísica cartesiana de su tiempo que fue reconocida por el mismo Malibranche. ${ }^{2}$ Entre todos los especialistas y traductores de este autor, poco se ha trabajado la analogía como un modo de conocimiento (Cfr., Kabitz 1974, Ritter 1853, Mahnke 1925, Friedmann 1944), es más, son de verdad, muy reducidas las empresas que han tocado el tema de la analogía en esta época historia (Siglo XVII) la cual Leibniz vive con gran intensidad (Cfr., Scheneiders 1994). Tratemos, por ende de entrar en el tema. En

\footnotetext{
${ }^{2}$ Una prueba de ellos es lo "basto" de su obra, recuperada y archivada en los Leibniz-Archiv y en el
} centro de estudios leibnicianos de Münster por citar algunos lugares. 
Leibniz la analogía se hace presente a lo largo de toda su obra (según Cassirer como una ley metafísica, 1902), donde la que más llama la atención es la que utiliza en la Teodicea de 1710, la armonía preestablecida, la cual, va apareciendo a lo largo del libro. En ella encontramos la sutil forma de conducirse de este autor al momento de hablar de Dios, la bondad y la maldad, considerando lo suficiente, después de su debate contra Spinoza, la ética como un medio para comprender la naturaleza del hombre y lo importante del conocimiento en la vida del mismo (Cfr., Orio de Miguel, 1988).

Por ejemplo, cuando él habla de esta categoría la utiliza para referir, ciertamente, una analogía, pues: “(...) como para explicar esta maravilla de la formación de los animales me valí de una armonía preestablecida, es decir, del mismo medio que había empleado para explicar otra maravilla, cuál es la correspondencia del alma con el cuerpo" (Teodicea, prefacio). En sí misma la armonía preestablecida determina un tipo de "sistema" como Leibniz le llama, siendo una proporción "bien ordenada" que refiere, sin duda, a una proposición categorial que determina el componente funcional del diagrama leibnizsiano. Así, "proposiciones de razón son las que surgen únicamente de las ideas o, lo que es igual, que nacen de un conjunto de definiciones que no deben su origen a los sentidos y que; por tanto, son hipotéticas, necesarias, eternas, como todas las proposiciones abstractas de la geometría, de la aritmética y la foronomía (cinemática)”. (Leibniz II, I, 2003).

La analogía, vista como un referente necesario de construcción explicativa (proposicioinal), surge no sólo por la necesidad de exposición, sino que, en este caso, representa un modo más de llegar a las operaciones simples. Este modo de arribar a la concreción de la demostración "armónica", el autor ocupa la diversidad y la identidad aludiendo que: "la armonía y la discordancia (...) consisten en la razón entre identidad y diversidad, ya que la armonía es la unidad en la multiplicidad, y es máxima en la mayor multiplicidad; y en especial, cuando algunos elementos aparentemente desordenados son súbitamente reducidos mediante cierta razón admirable al sumo orden". (Leibniz II, I, 2003). Ocupando la analogía (de proporción y Leibniz era buen lector de los medievales, de los cuales aprendió el arte combinatoria lluliana y su discusión con Escoto o con Pedro de Poznan e.g.,) llega a comentar que entre la unidad y la multiplicidad existe una relación proporcional (cierta razón 
admirable) para llegar al sumo orden (Cfr., Beuchot, intrd. 1986) que será la correspondencia entre la demostración y la definición a partir de la comprobación del esquema condicionante. Cabe destacar acá el uso de la analogía al momento de justificar la función del razonamiento, no sólo matemático, sino "de las cosas más naturales" para acceder a la armonía universal, a partir de ciertas leyes del razonamiento, pues: "lo único cierto es que nosotros percibimos, que percibimos coherentemente y que al percibir observamos ciertas reglas. Percibir coherentemente significa que se percibe de modo tal que se puede dar razón de todo y que todo se puede percibir. La existencia consiste en esto: en percibir observando ciertas leyes, pues, de lo contrario, todo sería como un sueño". ${ }^{3}$ (Leibniz II, I, 2003). La proporcionalidad radica, tanto en aquello de las "ciertas leyes" y lo percibido, pues la interpretación no es directa dada la inexactitud de la demostración. No así en cambio en la indicación a las verdades absolutamente primeras (que refieren a la armonía preestablecida como tales), de hecho "las verdades absolutamente primeras ${ }^{4}$ son las idénticas entre las verdades de razón y, entre las verdades de hecho, la siguiente (a partir de la cual todas las experiencias se pueden demostrar a priori), esto es, todo posible exige existir ${ }^{5}$ y, por tanto, llegaría a existir a menos que lo impida otra

\footnotetext{
${ }^{3}$ Castañeda sugiere que en este pasaje el pensamiento de Leibniz se halla en tensión entre dos sentidos: a) la interpretación fenomenista reductiva: según la cual un objeto existente es sólo una colección de contenidos perceptivos estructurados según leyes, b) la interpretación epistemológica, según la cual el criterio que poseemos para sostener o postular la existencia de cierto objeto es la sumisión de las apariencias del objeto en las mentes según leyes definidas. La tensión de resolverá más adelante en: c) la interpretación de la dependencia ontológica: un objeto no es sólo una colección de contenidos perceptivos, sino una realidad particular que está fuera del espíritu, aunque esa realidad necesariamente es una entidad que puede aparecer a perceptores y sus apariencias están gobernadas por leyes de modo tal que las leyes de la apariencia perceptiva son también leyes constitutivas de objetos. (Vid., nota I, en Leibniz. II, I, 2003).

4 "(...) la definición de la verdad es real. Es verdadero lo que se puede demostrar por medio de definiciones a partir de lo idéntico. Lo que se demuestra a partir de definiciones reales es absolutamente verdadero. Sólo pueden aducirse definiciones reales de aquellas nociones que nosotros percibimos inmediatamente, e.g., la extensión [de un cuerpo], la temperatura, cuando digo que es verdadero que existen. En efecto, se debe asignar, además, algo distinto a aquello mismo que percibimos confusamente. Las definiciones reales se pueden probar a posteriori, a saber, por experiencia. Que todo existente es posible se debe demostrar a partir de la definición de existencia”. (Leibniz. II, I, 2003).

5 "(...) todo posible exige existir, puede ser probada a posteriori si se admite que algo existe. En efecto, o bien todas las cosa existen y entonces todo posible exige existir hasta el punto de existir también, o bien algunas cosas no existen, y entonces debe darse razón de por qué algunas cosas existen en lugar de otras pero en este caso no puede darse otra razón que la razón general de la esencia, o sea, la posibilidad, si se admite que por su naturaleza es posible exigirle la existencia, y por cierto,
} 
cosa que también exija existir y que sea incompatible con la anterior. De ahí se sigue que siempre existe la combinación de cosas mediante la cual existe el mayor número". (Leibniz II, I, 2003). Entre verdades de razón y las de hecho, todo posible exige existir, es la analogía que utiliza el autor para ensayar su arte combinatoria. Por ejemplo, “(...) las primeras nociones de cuya combinación surgen las restantes son distintas y confusas. Son distintas las que se entienden por sí mismas, como la noción de 'ser'. Son confusas y sin embargo claras, las que se perciben por sí mismas [i.e.,] que no se puede explicar a otro si no es mostrándoselo. Pues aunque por su naturaleza sea resoluble (es decir analizable), puesto que tiene una causa, sin embargo no lo podemos describir y reconocer suficientemente mediante ninguna nota que se pueda tomar por separado, sino que se conoce sólo confusamente y por ello no admite una definición nominal. La definición nominal consiste en la enumeración de las notas o de los requisitos que son suficientes para distinguir una cosa de todas las demás. En efecto, si se buscan siempre los requisitos de los requisitos se habrá de llegar por último a las nociones primitivas que o bien carecen en absoluto de requisitos o bien nosotros podemos explicar de modo suficiente. Éste es el arte de tratar las nociones distintas." (Leibniz III, 2003).

Ésta tan nombrada y desarrollada definición nominal, sirve a Leibniz como analogía para distinguir las cosas de todas y cada una de las demás, a saber, utilizando la analogía de proporción, damos cuenta del funcionamiento del arte combinatoria (a diferencia de su lenguaje universal) que subraya la diferencia entre diversidad (multiplicidad de las cosas) y la unidad (la no variabilidad de la relación, aunque no de la demostración). En conclusión, cuando Leibniz echa mano de la analogía de la armonía preestablecida, nos presenta el entendimiento de la unidad y la multiplicidad, dadas las condiciones de demostración y combinación, sin la cual, sería complejo entender las definiciones nominales y la misma armonía preestablecida.

según la razón de posibilidad, o sea, según el grado de la esencia. Si en la naturaleza misma de la esencia no hubiera cierta inclinación a existir, nada existiría. Pues decir que algunas esencias poseen cierta inclinación, pero otras no, es decir algo sin razón (acá si a existencia fuera algo diferente de la exigencia, se seguiría que ella misma tiene cierta esencia, o sea, que se sobre agrega algo nuevo a las cosas. Se puede preguntar enseguida a cerca de esto si esa esencia existe y por qué existe ésta concreta más bien que aquélla), pues generalmente parece que la existencia se refiere a toda esencia del mismo modo". (Leibniz II, I, 2003). 
Asimismo, la analogía de proporción directa (a la que ya hemos aludido en las citas del autor) demuestra la relación existente entre lo uno y lo diverso, pues: "(...)la unidad en la multiplicidad no es otra cosa que la armonía, y como uno concuerda más con esto que con aquello, emana de allí el orden, del que proviene toda hermosura, y la hermosura despierta amor". (Leibniz VII., 2003).

Esta "condición nominal" la encontramos en Kant, sobre todo al momento de desarrollar la noción de analogías tanto de conocimiento como de experiencia.

\section{La analogía en Kant}

Sin lugar a dudas, Immanuel Kant (1724-1804) es uno de los pensadores más influyentes, no solo en la filosofía, sino en prácticamente todo el conocimiento universal. Gran deudor de David Hume, Kant trata de aportar varias disquisiciones que, a la fecha, nos permiten el entendimiento de su manera de pensar y de desarrollar un sistema filosófico. Se le puede aplicar la frase de que: “ el escritor popular despierta en nosotros la creencia de que lo presentado se comporta realmente (wirklich) así, pero tampoco aporta nada más, pues nos hace sentir la verdad de aquella proposición, pero sin conducir a la absoluta certeza. El sentimiento puede, no obstante, enseñarnos lo que es, pero jamás lo que tiene que ser. El escritor filosófico [científico] eleva aquella creencia a convicción, pues prueba con razones indudables que se comporta así necesariamente (notwendig)". (Fichte, 1998). Kant verdaderamente es un escritor filosófico, pues nos conduce a la elevación del espíritu y a la crítica necesaria para ir estructurando el conocimiento.

Por lo basto y difícil del pensamiento kantiano, sólo nos limitaremos, como ya hicimos mención, a algunos textos. Trabajaremos primero el parágrafo 58, luego un apartado de la Crítica. Al final del parágrafo 57 Kant menciona que “(...) si yo digo que necesitamos concebir el mundo como si fuese la obra de un entendimiento y una voluntad superiores, no digo, en realidad más que: como se relaciona un reloj, un barco o un regimiento con un relojero, un ingeniero o un combatiente, así se relaciona el mundo con los sentidos (o lo que constituye los fundamentos de este conjunto de fenómenos), con lo 
desconocido que yo, por tanto, no conozco, según lo que es en sí mismo, sino según lo que es para mí, a saber, en relación al mundo del cual soy parte”.

A esto el autor llama un conocimiento según la analogía. Empero, a diferencia de Leibniz que veíamos que ocupaba la analogía de proporción al hablar de armonía preestablecida, Kant hace uso de la analogía por atribución indirecta o figurativa; su primer analogado es la relación con el mundo y sus analogados subsecuentes son las relaciones de los sentidos con los fenómenos. Cabe destacar que la analogía o el conocimiento por analogía en este ejemplo, nos muestra que las supuestas relaciones con el mundo no parten del hecho mismo de percatarnos de los fenómenos a través de los sentidos, sino que nos damos cuenta de que pertenecemos al mundo y que nos relacionamos con este. No sólo "existen los fenómenos en sí" sino que, para que yo pueda pensarlos (sentirlos) debo de estar en el mundo. Ya en el siguiente parágrafo notamos esto: "un conocimiento tal es el conocimiento según la analogía, el cual no significa, como se entiende generalmente esta palabra, una semejanza incompleta de dos cosas, sino una semejanza completa de dos relaciones entre cosas completamente desemejantes. Por medio de esta analogía obtenemos, pues, un concepto del ser supremo suficiente para nosotros, aunque le hayamos desposeído de todo lo que podía determinarle pura y simplemente y it en sí mismo; pues le determinamos con respecto al mundo, y, por consiguiente, a nosotros, y tampoco es necesario más" (58).

Resaltemos la frase de que aquello que se ha pensado como analogía no es una semejanza incompleta de dos cosas, sino una semejanza completa de dos relaciones entre cosas completamente desemejantes. La analogía no podría ser una semejanza incompleta de dos cosas, pues no existe una semejanza incompleta, si por semejanza entendemos, como lo hace Kant, la relación entre el reloj y el relojero, el barco con el ingeniero o el comandante y el regimiento, pues la relación debe de ser completa. No entenderíamos la relación sin su analogado primero. Es más, no se nos está permitida la semejanza incompleta porque sería, en sí misma, una contradicción de sentido. Si aceptamos el it primer analogado (el relojero, el ingeniero o el comandante en cada uno de los cosas) que son los que nos otorgan los analogados it segundos (el reloj, el barco o el regimiento) entonces, la relación de semejanza es completa, diga- 
mos, a condición de que exista, la correspondencia del mundo de los sentidos con lo desconocido y así sucesivamente.

Atribuimos la "propiedad figurativa" de las relaciones semejantes, porque estamos obligados a aceptar el primer analogado que, según este autor, es el que nos permite conocer no lo que la cosa es en sí, sino lo que es para mí, que no es otra cosa que la relación al mundo del cual soy parte. Más aún, en el pié de página de ese mismo parágrafo se lee "en este caso está la analogía entre las relaciones jurídicas (lo universal como condición de la ley y lo particular del caso por sancionar por ejemplo) de las acciones humanas y las relaciones mecánicas (velocidad, atracción, desplazamiento, fricción, etcétera) de las fuerzas en movimiento, no puedo nunca hacer algo con respecto a otro sin concederle el derecho de hacer precisamente lo mismo conmigo bajo las mismas condiciones, del mismo modo que cuerpo alguno puede obrar sobre otro con su fuerza en movimiento, sin causar por esto que el otro reobre igualmente sobre él” [n. al p. par. 58, pag., edic. 151]. Es clara la analogía de atribución figurativa, tanto de lo que se considera como la it relación entre lo semejante, como de lo que se conoce de los fenómenos a través de los sentidos. Lo que no queda muy claro es el criterio de tal aceptación de la relación. Kant mencionará que este criterio será exactamente en el límite de todo uso lícito de la razón. Ahora bien, si ya quedó claro por qué es una incongruencia pensar una semejanza incompleta, vayamos a la definición dada por el autor.

Él comenta que la analogía será una semejanza completa de dos relaciones entre cosas completamente desemejantes. Pero ¿̇cómo se da la semejanza completa? Se da por el uso de la atribución a la relación de semejanza (no a la completud) porque necesitamos que los dos objetos estén incluidos en la semejanza. El ejemplo más claro (que lo podemos encontrar en Leibniz antes que en Kant) de aquello que el juicio es, la capacidad de pensar el particular contenido en el universal. Acá hay semejanza, según Kant, completa, pues la relación de atribución se da por la aceptación del primer analogado (el universal) que debe de incluir (derivar) al segundo analogado (lo particular). En el mismo lugar se comenta "por ejemplo, del mismo modo que se relaciona la producción de la felicidad de los niños $=\mathrm{a}$, con el amor de los padres $=\mathrm{b}$, se relaciona la salud del género humano $=\mathrm{c}$, con lo desconocido en Dios $=\mathrm{x}$, a lo cual llamamos amor; no como si éste tuviese la menor semejanza con alguna 
inclinación humana, sino porque, sus relaciones con el mundo, las podemos establecer como semejantes a las que mantienen las cosas del mundo entre sí" [n. al p. par. 58, pag., edic. 151]. Se percibe aún con mayor fuerza la atribución y la analogía "completa". Lo que no "cuadra" es la segunda parte de la definición, aquello de las dos relaciones entre cosas completamente desemejantes.

Pero ¿de dónde surge tal duplicidad de relaciones? y ¿cómo se da la relación entre lo completamente desemejantes? La primera respuesta la da Kant en el desarrollo del parágrafo, explicando que las dos relaciones son la causalidad por la razón y la relación al mundo de los sentidos, los cuales dan la pauta para el desarrollo del conocimiento según la analogía. A la segunda pregunta, la relación entre lo completamente desemejante, lo menciona más delante, cuando dice que "se considerará solamente la causa de la forma de la razón, que se encuentra por todas partes en el mundo, y se atribuye, ciertamente, la razón al ser supremo, en tanto que contiene el fundamento de esta forma de la razón, pero solamente it según la analogía, esto es, en tanto que esta expresión sólo indica la relación que, la causa suprema, desconocida para nosotros, tiene con el mundo, para determinar en él todo, en el más alto grado, según la razón" (58). Es decir, sólo indica la relación entre lo completamente semejante y según la analogía, lo cual demuestra que sería impensable una estructura tal de incompletud, en el sentido de la misma razón. Lo it entre completamente desemejante, alude, en todo caso, a la relación (sin contenido, a saber figurativa) de lo completamente semejante.

En it Was heisst: sich im Denken orientieren? de 1786, Kant vuelve a ocupar la analogía de atribución (indirecta o figurativa) para proponer una manera de orientarnos en el pensamiento, expresando que "aunque podamos elevar a lo alto nuestros conceptos y, con ello, abstraer[nos] de la sensibilidad, siempre dependen de ellos representaciones it figurativas, cuya determinación propia consiste en volver aptos para elit uso de la experiencia a los conceptos, que, por lo demás, no derivan de la experiencia"(ver. 1999, p. 165). Las mentadas representaciones figurativas no generan conocimiento, sino sólo nos permiten percatarnos de las relaciones en el mundo, que estamos en el mundo, como lo mencionó más arriba. Y de hecho, nos relacionamos y estamos en el mundo de manera analógica, buscamos siempre la "figuración", es decir, la 
representación simbólica con la misma realidad y el mundo natural. A esto hay que agregar que Kant utiliza en la Crítica de la razón pura la analogía como Verbindung, cuando justifica el principio general en la necesaria unidad de apercepción con respecto a toda conciencia empírica. Comenta el autor: "el principio general de las tres analogías (permanencia, sucesión y simultaneidad) se basa en la necesaria it unidad de apercepción con respecto a toda conciencia empírica (de la percepción) posible it en todo tiempo. Se basa, pues $(. .$.$) en la unidad sintética de todos los fenómenos, por lo que a su re-$ lación (Beziehung) temporal concierne. En efecto, la apercepción originaria, se refiere al sentido interno (al conjunto de todas las representaciones). Se refiere a priori a la forma del mismo, es decir, a la relación temporal de la conciencia empírica diversa. Toda esta diversidad tiene que ser unificada en la apercepción originaria de acuerdo con sus relaciones de tiempo" (2000, B 220). Esta Verbindung se da con base en el primer analogado que es el tiempo y las tres analogías (permanencia, sucesión y simultaneidad) se usan como analogados secuenciales. Por ello mismo la analogía de atribución toma acá un doble papel y significado, el primer analogado es un Beziehung, mientras los tres analogados secuenciales son Verbindung. Más fácil, relación y conexión se dan como modos de la seriación atributiva entre constitución explicativa aparente del fenómeno y la aprehensión condicionada del mismo.

Kant explica lo que decimos con una seriación atributiva: "las analogías significan en la filosofía algo muy distinto de lo que representan en las matemáticas. En éstas constituyen fórmulas que expresan la igualdad de dos relaciones cuantitativas y son siempre it constitutivas; de forma que, dados tres miembros de la proporción, se da también el cuarto, es decir, puedo construirlo. En filosofía, en cambio, la analogía no es la igualdad de dos relaciones it cuantitativas, sino la de dos relaciones cualitativas. Es una igualdad en la que, dados tres miembros, puedo simplemente conocer e indicar a priori la relación con un cuarto miembro, pero no conocer it este cuarto miembro directamente" (A 180). Y acá mismo nos da la pauta para mencionar la analogía por atribución indirecta, pues esta no es constitutiva sino que, según el autor, recrea la condición de aplicación del principio general, es decir, la unidad, que es cualitativa. En conclusión, Kant utiliza la analogía de atribución indirecta para señalar las causas por las cuales podemos construir marcos explicativos de aquello que, 
en su referencialidad, sería imposible el entendimiento de ciertos elementos de la naturaleza. ${ }^{6}$ Comenta el autor: "a la hora de señalar una causa, no podemos hallar un procedimiento más seguro que el de la analogía con esos productos dotados de finalidad. Son los únicos cuya causa y cuyo modo de operar conocemos perfectamente. La razón no podría justificarse ante sí misma si quiera pasar desde una causalidad que conoce a sus causas explicativas oscuras e indemostrables" (A 626, B 654).

Que la analogía sea, para Kant un procedimiento nos posibilita entender el fundamento de atribución de la misma demostración que es, en última instancia, sólo la mera contingencia de la forma, más no de la materia.

\section{Conclusión}

Como dimos cuenta, la analogía (tanto de proporción directa como de atribución indirecta) nos posibilitan entender que, para poder acercarnos al mundo, es necesario contemplar la importancia de esta categoría como fundamento en la explicación interpretativa. Por ello, lo mencionábamos más arriba, la analogía es autorreferente por el simple hecho de que ella es un fundamento límite, fragmentario, pero suficiente, que nos permite construir conocimiento, más no es lo construido ni es lo por construir. Pues a la pregunta guía de este ensayo: la analogía, puede llegar a determinar la construcción del conocimiento o simplemente es un "modelo" para interpretar lo ya construido? La respuesta es que para Leibniz si genera ceritas condiciones para la construcción del conocimiento, mientras que para Kant la analogía sólo servirá para identificar lo que se puede comprender y lo que no se puede comprender.

No obstante, si pensamos un poco en esto, la referencia a sí misma le da sentido a la analogía, pues sin sentido y sin referencia una hermenéutica o cualquier otra figura no tendría razón de ser, puesto que la interpretación sin sentido no es comprensible y sin referente no podría ser explicada.

\footnotetext{
${ }^{6}$ Así lo expresa cuando: "de la analogía que existe entre algunos productos naturales y lo que es resultado del arte humano cuando la razón violenta la naturaleza y la obliga a no seguir sus fines, sino a doblegarse a los nuestros, infiere esta misma razón (apoyándose en la analogía existente entre esos productos naturales y casas, barcos y relojes) que hay en la naturaleza una causalidad de este mismo tipo; es decir, al derivar la interna posibilidad de una naturaleza que actúa por si mismo (frewirkenden Natur) [la cual hace posible todo arte y quizá la razón misma] de otro arte, de un arte sobrehumano, la razón infiere que la naturaleza se basa en el entendimiento y la voluntad" [A 626, B 654]
} 
Tanto Leibniz como Kant nos muestran la condición de posibilidad para acercarnos a los límites interpretativos de relación, proporcionalmente hablando, a algunas de las intencionalidades de los autores que son, de una manera u otra, los caminos para la comprensión y el análisis. Como lo menciona Beuchot sobre este tema: "[la analogía] alcanza la suficiente claridad y distinción dentro de lo que de suyo tiende a la oscuridad y a la confusión” (2010). A partir de una interpretación analógica, autorreferencial, podremos, de forma reducida, pero insistimos, suficiente y necesaria, acercarnos a los sentidos y a las referencias del conocer humano que es, sin lugar a dudas, la tarea más artística del filósofo.

\section{Referencias}

Aristóteles, 2001, Física, Bibliotheca Scr. Grae. et Rom., UNAM, México.

—, Metafísica, 1998. Biblioteca Clásica Gredos, Madrid,. trad. Tomás Calvo.

Beuchot, M. 1986, Introducción al libro de Leibniz, Discusión metafísica sobre el principio de individuación, UNAM, México.

— 2010, Somera exposición de la Hermenéutica analógica, en Blanco. R. (comp). Praxis de la hermenéutica analógica, Torers y Asoc., México.

— 2002, Perfiles esenciales de la hermenéutica, UNAM, México.

——, 2009, Tratado de hermenéutica analógica. Hacia un nuevo modelo de interpretación, ed., Ítaca-UANM, México.

Cassirer, E, 1962, Leibniz'System in seinen Wissenschaftlichen Grundlangen, Olms, Verlag, Hildesheim- Deuch-Eng.

Fichte, J. G., 1998, Filosofía y estética, Collecció estética e crítica, Universidad de Valencia, Valencia.

Friedmann, G., 1944, Leibniz et Spinoza, Gallimard, París.

Galison, F., 2000, "Bacon, Galileo and Descartes on Imagination and Analogy", en ISIS, vol. 75, num., 277.

Kabitz., W., 1974, Die Philosophie des jungen Leibniz, edic., 1909, Nachdruk der Ausgabe Heidelberg, ed., Olms Verlag, Hildesheim, Deuch-Eng.

Kant, I, 2000, Crítica de la razón pura, ed., Alfaguara, España.

— 1999, En defensa de la Ilustración, Alba Edit., Barcelona.

, 1999, Prolegómenos a toda metafísica del futuro, Losada, Buenos Aires.

Leibniz, G. W., 2003, Escritos filosóficos, Ed. Mínimo Tránsito, Madrid.

_- 2001, Teodicea, en Obras completas, Madrid.

—, 2004, Tratados fundamentales. Discurso de metafísica, Losada, Buenos Aires.

Mahke, D., 1964, Leibnizens Synthese von Universalmathematik und Individualmenthaphysik, Halle 1925, fasc. Edic. Neudruck, Frommann Verlag, Stuttgart. 
Mendoza, R., 2008, "Bases epistemológicas de la comunicación", en Conde G. N. (comp). Contornos de la hermenéutica analógica, Torres y Asoc., México.

, 2010, Sobre la sutileza, en Blanco., R. Praxis de la Hermenéutica analógica, Torres y Asoc., México.

Orio de Miguel, B., 1988, "El principio de analogía en Leibniz", en Quaderns de Filosofia, Enrahonar, 14, Universidad Autónoma de Barcelona, pp. 33-61.

Paredes., M., 2007, Teorías de la intencionalidad, Síntesis, Madrid.

Ricoeur, P., 2001, La metáfora viva, Ediciones Cristianas, Madrid.

Ritter, H., 1983, Geschichte der Philosophie, Hamburgo, 1853, Zwölfter Teil, Siebentes Buch, Edic. Zweites Kapitel, Hamburgo.

Scheneiders, W., 1984, "Harmonia Universalis", en Stud. Leibniz, Band 16, Leibniz Werk und Wirkung, Edic., Z. Kapitel.

Teofrasto, 1991, Algunas cuestiones de metafísica, Anthropos, Barcelona.

Recibido el 2 de Marzo de 2011

Aceptado el 20 de Mayo de 2011 